\title{
Isolated Extramedullary Relapse in a Case of Acute Myeloid Leukemia Following Allogeneic Stem Cell Transplantation
}

\author{
Raafat Abdel-Malek ${ }^{1}$, Rehab Sayed ${ }^{2}$, Shady E. Anis ${ }^{3}$ \\ ${ }^{1}$ Clinical Oncology Department, Kasr Al-Ainy School of Medicine, Cairo University, Cairo, Egypt; \\ ${ }^{2}$ Clinical Oncology Department, Arab Organization for Industry Hospital, Cairo, Egypt; ${ }^{3}$ Pathology \\ Department, Kasr Al-Ainy School of Medicine, Cairo University, Cairo, Egypt
}

\begin{abstract}
Background: Acute myeloid leukemia (AML) is the commonest acute leukemia in adults. Allogeneic stem cell transplantation (ASCT) is a curative option for a subset of these patients.

Case Presentation: We report the case of a 36 years old female patient who presented in April 2014 with pancytopenia. Investigations revealed that she had AML (M6). She achieved complete remission with induction chemotherapy which was followed by four cycles of consolidation chemotherapy until a human leukocyte antigen-matched donor was available. She underwent ASCT in January 2015. After 14 months, in March 2016, she presented with left breast and right parapharyngeal masses. Histopathological examination of the excised mass showed infiltration with myeloid cells and the bone marrow was normocellular without leukemic infiltration. She received radiotherapy to the affected breast and the cervical region followed by chemotherapy with good response.

Conclusion: AML relapse following ASCT may be in the form of isolated extramedullary disease. Further research is needed to optimize the management of these cases.
\end{abstract}

Keywords: Acute myeloid leukemia, Allogeneic stem cell transplant, Extramedullary relapse Corresponding author: Raafat Abdel-Malek, MD; Clinical Oncology Department, Kasr Al-Ainy School of Medicine, Cairo University, Cairo, Egypt; Email: Raafat.malek@kasralainy.edu.eg

Submitted: 5-December-2017, Revised: 31-December-2017, Accepted: 8-January-2018, Published online: 5-April-2018

\section{INTRODUCTION}

Acute myeloid leukemia (AML) is the commonest form of acute leukemia among adults. Patients with a high risk of relapse are considered for allogeneic stem cell transplantation (ASCT).

Extramedullary relapse (EMR) after SCT for AML is under-reported and usually occurs late. Among longterm survivors, incidence may reach up to $20 \%{ }^{1-4}$. EMR without coexisting leukemic marrow involvement may occur because the graft-versus-leukemia (GVL) effect is more pronounced in the marrow than at extramedullary sites. Extramedullary relapse has been reported up to 10 years post-transplant ${ }^{2}$. Extramedullary relapse can involve many sites including the soft tissue, bone, skin, central nervous system, breast, nasopharynx, paranasal sinuses, intra-abdominal or pelvic organs as well as peritoneal and pleural cavities.

\section{CASE PRESENTATION}

We report the case of a 36 years-old female who presented in April 2014 with persistent easy fatigability. Complete blood picture was done and revealed pancytopenia. Bone marrow aspiration with flow cytometry confirmed the diagnosis of AML (M6).

She started induction treatment in May 2014 with $7+3$ protocol (cytarabine $100 \mathrm{mg} / \mathrm{m}^{2}$ continuous infusion for [days 1 to 7] plus mitoxantrone $12 \mathrm{mg} / \mathrm{m}^{2}$ [days 1 to $3]$ ). Bone marrow examination on day 28 revealed $6 \%$ blasts. Consequently, re-induction was attempted using high dose cytarabine $2 \mathrm{~g} / \mathrm{m}^{2}$ plus mitoxantrone $12 \mathrm{mg} / \mathrm{m}^{2}$ for 3 days which resulted in complete remission as evidenced by bone marrow examination (3\%) in June 2014. This was followed by consolidation with 2 cycles of cytarabine $1 \mathrm{gm} / \mathrm{m}^{2}$ plus etoposide $100 \mathrm{mg} /{ }^{2}$ for 3 days then 2 cycles of high dose cytarabine $2 \mathrm{~g} / \mathrm{m}^{2}$ plus mitoxantrone $12 \mathrm{mg} / \mathrm{m}^{2}$ for 3 days. Consolidation continued until October 2014 when a human leukocyte antigen - matched donor was available. She underwent ASCT in January 2015 with smooth recovery apart from chronic skin graft-versus-host disease (GVHD).

In March 2016, she developed a left breast hard mobile mass showing BIRADs $4 \mathrm{c}$ on sonomammography. At that time, she refused biopsy. Six months later, she presented with rapid increase in the size of the breast mass and new onset of dysphagia. Positron emission tomography-computed tomography (PET-CT) scan was done in October 2016 revealing left breast mass $(10 \times 10 \mathrm{~cm})$ as well as right parapharyngeal mass $(7 \times 7 \mathrm{~cm})$. Bone marrow examination was free of leukemic infiltration.

As biopsy was inconclusive, excision of the breast mass was done in November 2016 and histopathological examination showed infiltration by myeloid cells. The patient received radiotherapy to the left breast as well as bilateral cervical region 40 Gy divided on 20 fractions in 4 weeks followed by 3 cycles of high dose cytarabine 1.5 $\mathrm{g} / \mathrm{m}^{2}$ for 3 days which ended in April 2017 with 
regression of parapharyngeal mass down to $3 \times 3 \mathrm{~cm}$ and the patient was asymptomatic.

Three months later, the patient presented with right cervical mass $(12 \times 10 \mathrm{~cm})$ associated with progressive dysphagia and right otalgia. PET-CT scan showed activity at the right parapharyngeal region only. Biopsy from the parapharyngeal mass revealed neoplastic infiltrate, positive for myeloperoxidase, compatible with myeloid leukemic infiltrate (figure 1). Bone marrow examination revealed normocellular marrow with trilineage hematopoiesis and no leukemic infiltration (blasts counted for $1 \%$ of all nucleated marrow cells).

The patient started palliative chemotherapy with cytarabine $1 \mathrm{~g} / \mathrm{m}^{2}$ and etoposide $100 \mathrm{mg} / \mathrm{m}^{2}$ for 3 days to be recycled every 3-4 weeks. She received two cycles with good tolerance and marked regression of the right parapharyngeal cervical mass after the first cycle.

\section{DISCUSSION}

Continuous complete remission of AML after allogeneic bone marrow transplantation is due to combined effects of high-dose chemotherapy and graftversus-leukemia effect ${ }^{5}$. The graft-versus-leukemia effect is effective in preventing relapse in the bone marrow only with minimal effect on extramedullary sites.

Bone marrow relapse and EMR differ in their manifestations. The median time from ASCT to EMR is 10-17 months, which is longer than that from ASCT to bone marrow relapse ( 3 to 7 months) ${ }^{1,3}$. Extramedullary relapse occurs more commonly in patients with chronic GVHD ${ }^{6}$. Due to better patient support and longer life span post-transplant, the incidence of EMR is expected to rise over time and recurrent extramedullary leukemic relapses are likely to become more common.

Extramedullary relapse following ASCT has poor prognosis and is difficult to manage with no standard treatment $3,4,6,8$. Induction chemotherapy with localized radiotherapy to the involved site has been used. However, patients treated with high-dose chemotherapy are usually under immunosuppressant drugs to minimize GVHD; thus, they have high risk of toxicity from further treatment.

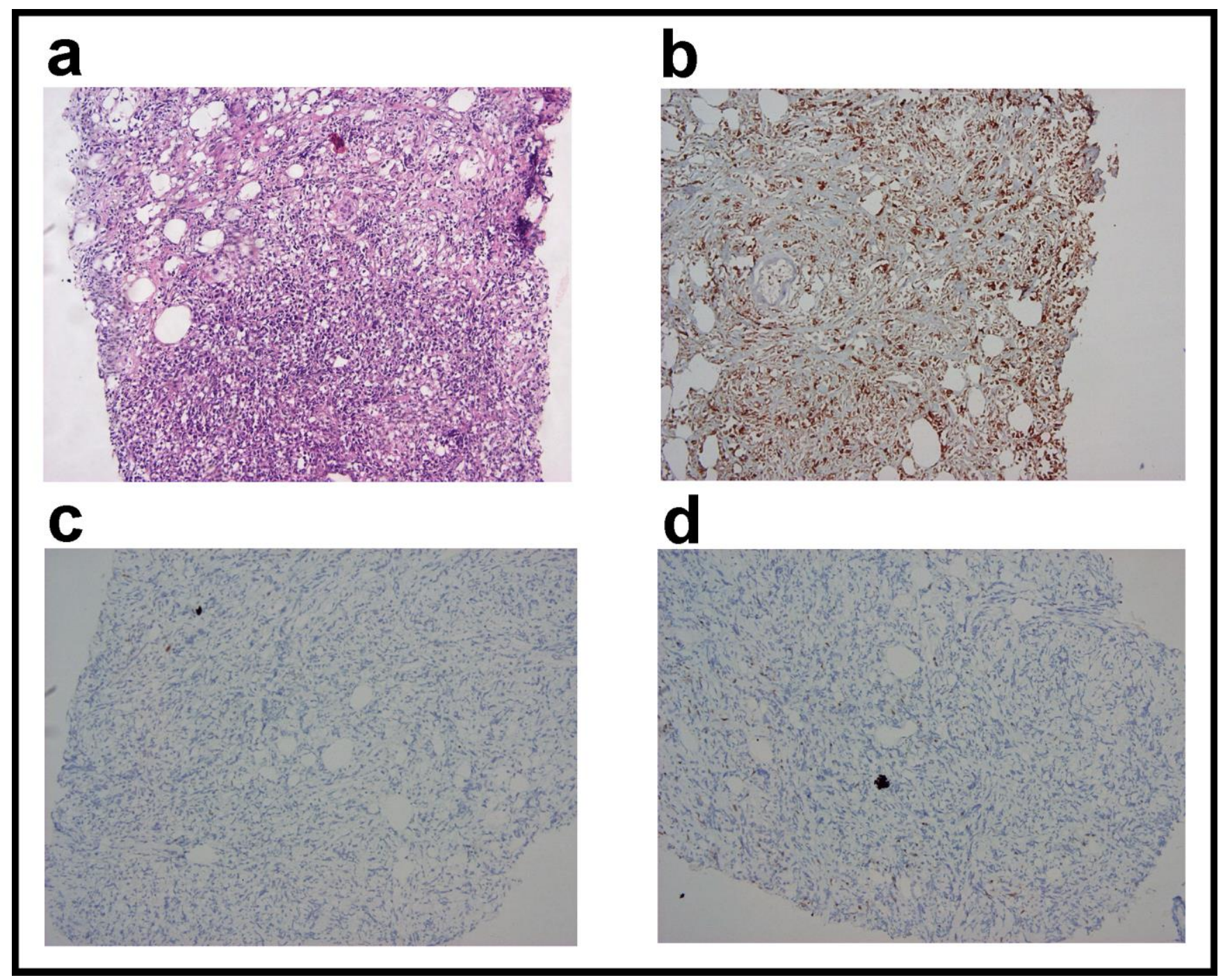

Figure 1. Hematoxylin and eosin and immunohistochemistry staining of biopsy from the parapharyngeal mass. a) Hematoxylin \& eosin b) Myeloperoxidase c) CD20 d) CD3. 
In our case, earlier suspected diagnosis of this condition as patient had chronic GVHD as well as earlier treatment with surgery or higher dose of radiotherapy could have given us better local control.

Due to lack of effective treatment with systemic chemotherapy for this condition, novel approaches are needed with a better understanding of the molecular genetics and risk factors that predispose individuals to developing EMR after ASCT. Therapies aiming at routing the graft-versus-leukemia effect to extramedullary tissues might improve the outcome. Use of novel immunotherapy drugs such as anti-PD1 / PDL1 may stimulate the immune cells to kill antigenexpressing cancer cells in soft tissue but also may increase GVHD necessitating further clinical trials.

\section{REFERENCES}

1. Harris AC, Kitko CL, Couriel DR, et al. Extramedullary relapse of acute myeloid leukemia following allogeneic hematopoietic stem cell transplantation: incidence, risk factors and outcomes. Haematologica. 2013; 98(2): 179184.

2. Cunningham I. Extramedullary sites of leukemia relapse after transplant. Leuk Lymphoma. 2006; 47(9): 17541767.
3. Lee KH, Lee JH, Choi SJ, et al. Bone marrow vs extramedullary relapse of acute leukemia after allogeneic hemopoietic cell transplant: risk factors and clinical course. Bone Marrow Transplant. 2003; 32(8): 835-842.

4. Koc Y, Miller KB, Schenkein DP, Daoust P, Sprague K, Berkman E.. Extramedullary tumors of myeloid blasts in adults as a pattern of relapse following allogeneic bone marrow transplantation. Cancer. 1999; 85(3): 608-615.

5. Horowitz MM, Gale RP, Sondel PM et al. Graft-versusleukemia reactions after bone marrow transplantation. Blood. 1990; 75(3): 555-562.

6. Chong G, Byrnes G, Szer J, Grigg A. Extramedullary relapse after allogeneic bone marrow transplantation for haematological malignancy. Bone Marrow Transplant. 2000; 26(9): 1011-1015.

7. Shem-Tov N, Saraceni F, Danylesko I, et al. Isolated extramedullary relapse of acute leukemia after allogeneic stem cell transplantation: different kinetics and better prognosis than systemic relapse. Biol Blood Marrow Transplant. 2017; 23(7): 1087-1094.

8. Singhal S, Powles R, Kulkarni S, Treleaven J, Saso R, Mehta J. Long-term follow-up of relapsed acute leukemia treated with immunotherapy after allogeneic transplantation: The inseparability of graft-versus-host disease and graft-versus-leukemia, and the problem of extramedullary relapse. Leuk Lymphoma. 1999; 32(5-6): 505-512. 